\title{
SIRT3 SNPs validation in 640 individuals, functional analyses and new insights into SIRT3 stability
}

\author{
CHRISTIAN-LARS DRANSFELD ${ }^{1}$, HAMED ALBORZINIA ${ }^{2}$, STEFAN WÖLFL $^{2}$ and ULRICH MAHLKNECHT ${ }^{1}$ \\ ${ }^{1}$ Saarland University Medical Center, Department of Internal Medicine, Division of Immunotherapy and Gene Therapy, \\ José Carreras Research Center, Kirrberger Strasse, Bldg. 45.3, D-66421 Homburg/Saar; ${ }^{2}$ University of Heidelberg, \\ Department of Pharmacy and Molecular Biotechnology, Im Neuenheimer Feld 364, D-69120 Heidelberg, Germany
}

Received October 20, 2009; Accepted December 14, 2009

DOI: 10.3892/ijo_00000574

\begin{abstract}
Sirtuins are critical players within multiple cellular pathways such as stress response, apoptosis and energy metabolism. They are associated with metabolic and degenerative diseases, the pathogenesis of cancer and are key elements in the regulation of cellular life span. From within the 7 known human sirtuins, SIRT3 recently stepped out of the shadow of SIRT1 showing strong effects on stress response, apoptosis, cell cycle and energy metabolism, mimicking effects of caloric restriction. We have identified two non-synonymous human SIRT3 SNPs and evaluated their impact on SIRT3 activity and stability. We assessed their influence on cellular energy metabolism in relation to SIRT1 and identified SIRT3 to increase cellular respiration by $80 \%$ when compared to SIRT1, which increased cellular respiration by only $30 \%$.
\end{abstract}

\section{Introduction}

The sirtuins represent a family of enzymes that contain phylogenetically highly conserved catalytic domains that catalyze $\mathrm{NAD}^{+}$-dependent protein deacetylation and/or ADPribosylation. In numerous model organisms they have been described to be important regulators of longevity and elements that are essential in the regulation of transcriptional silencing, apoptosis and cellular stress response. Also, they are known to be essential in the regulation of cellular metabolism, the pathogenesis of cancer and degenerative diseases and thus, they have recently gained significant attention as molecular targets in the development of novel epigenetic therapeutics (1-4). To date, seven human sirtuins have been reported out of which SIRT1 has been the most intensively studied (2-4).

Correspondence to: Dr Ulrich Mahlknecht, Saarland University Medical Center, Department of Internal Medicine, Division of Immunotherapy and Gene Therapy, José Carreras Research Center, Kirrberger Strasse, Bldg. 45.3, D-66421 Homburg/Saar, Germany E-mail: ulrich.mahlknecht@uks.eu

Key words: SIRT3, single nucleotide polymorphism, energy metabolism, biosensor chip
More recently, SIRT3, the major mitochondrial deacetylase came to the fore, presenting itself with a major impact on energy metabolism, stress response and with distinct effects on apoptosis and cell cycle regulation (Fig. 1B, C, E and F) (5-12). In analogy to known SIRT1 effects within the cell nucleus, SIRT3 interacts with mitochondrial substrate isoforms of (cytosolic or nuclear) SIRT1 substrates such as Acetyl CoA-synthetase and FOXO3a (Fig. 1B and D) $(11,13)$. Also, a recent proteomic analysis identified $20 \%$ of mitochondrial proteins to be acetylated; including many metabolic enzymes and longevity regulators, all of which represent putative substrates for SIRT3, since SIRT3 knockout was shown to go along with global mitochondrial hyperacetylation $(5,14)$. SIRT3 is a mitochondrial protein, which is being activated in the mitochondrial matrix through cleavage to the $28 \mathrm{kDa}$ isoform by the matrix processing peptidase (MPP) (Fig. 1A) (15). Interestingly, SIRT3 activates the nuclear transcription of mitochondria related genes such as UCP1 (uncoupling protein 1), PGC-1 $\alpha$ (peroxisome proliferator-activated receptor $\gamma$ ) and COX (cytochrome oxidase) IV and V, which activate CREB (7). Similar to the class I and II HDACs, the sirtuins are differentially expressed in different types of cancer and we have been able to show that SIRT1 and SIRT3 are overexpressed in acute myeloid leukaemia (unpublished data) $(16,17)$. SIRT3 overexpression increases cell respiration, reduces the mitochondrial membrane potential and the level of reactive oxygen species, thus mimicking the effects of calorie restriction on cell metabolism and senescence (7).

The more recent literature on SIRT3 gives inconsistent information on the existence and impact of SIRT3 SNPs, e.g., on the relevance of SIRT3 SNPs in conjunction with survival in the elderly (18). In order to further elucidate the relevance of putative SIRT3 SNPs, we focussed our analyses on coding, non-synonymous SNPs (nSNPs), since these predicted an alteration in the SIRT3 amino acid sequence. The identification and validation of SIRT3 nSNPs could pave the way for further associative studies linking sirtuin biology with metabolic disease, organ degeneration and cancer.

In our study we validated two out of five putative nonsynonymous coding SIRT3 SNPs (R80W and V208I) and evaluated their impact on SIRT3 enzymatic activity and protein stability in relation to wtSIRT3 and wtSIRT1. Interestingly, 


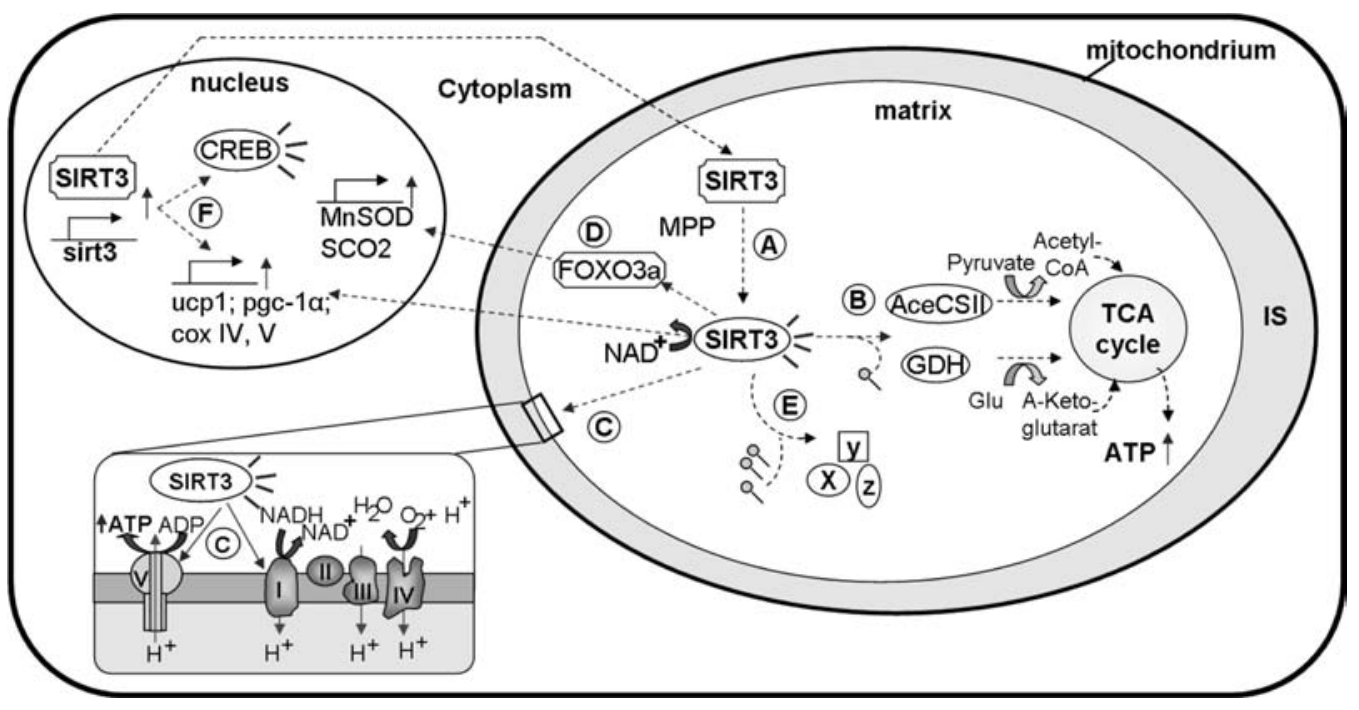

Figure 1. Overview on known SIRT3 interaction partners in energy metabolism. IS, intermembrane space. (A) SIRT3 processing via MPP; (B) SIRT3 deacetylates: AceCSII, GDH, Ku-70; (C) subunits of complex I and V; (D) FOXO3a; (E) 20\% of mitochondrial proteins are acetylated, presenting putative SIRT3 substrates; (F) SIRT3 induces CREB and expression of ucp1, pgc-1 $\alpha$, complex IV and V.

Table I. Putative SIRT3 nSNPs.

\begin{tabular}{lllccc}
\hline dbSNP rs no. & AB assay no. & $\begin{array}{c}\text { Sequence [Vic/Fam] } \\
\text { (sense/anti sense }=\text { s/as) }\end{array}$ & Exon & Codon-position & AS-exchange \\
\hline rs28365927 & C_25753927_10 & CTCC[A/G]GAAT (as) & 1 & 1 & R80W \\
rs11246020 & C_25754220_10 & GTGA[C/T]GTTG (as) & 3 & 3 & 1 \\
rs1734492 & C_7503249_10 & AGCC[C/G]GAGA (as) & 3 & 2 & V208I \\
rs1734491 & C_7503273_10 & GCAG[G/T]TGGC (as) & 4 & 1 & T255N \\
rs3020901 & SIRT3_0901-0901 & ACGC[C/T]GTGA (as) & 6 & G369S \\
rs7927733 & SIRT3_5927-5927 & TTTTC[A/C]GGTG (s) & Intron 5 & Splice site \\
\hline
\end{tabular}

despite the growing number of SIRT3 publications and its manifold cellular functions, SIRT3 protein stability has not been addressed so far and is one of the topics in the study presented herein.

\section{Materials and methods}

Genomic DNA isolation. Genomic DNA from 640 healthy Caucasian donors was extracted from $5 \mathrm{ml}$ peripheral blood by salting out with subsequent quantification on a Nanodrop ND-1000 Spectrophotometer (Peqlab Biotechnologie GmbH, Erlangen, Germany).

In silico analyses. In silico analyses were performed on the dbSNP (NIH) (19), HapMap (20), and ABI (Applied Biosystems) databases (21). Analyses were focused on coding, non-synonymous SIRT3 (AF083108) SNPs. Previously validated SNPs from the literature were also included.

Allelic discrimination. Allele specific SNP-assays were performed by TaqMan-PCR on an ABI Prism 7700 Sequence Detection System (ABI, Darmstadt, Germany) in accordance with the instructions provided by the manufacturer (Table I).
Cell culture, transfections. Hek293T and HeLa (DSMZ, Braunschweig) and H-JR-Mito-HeLa cells (Marinpharm, Luckenwalde), were cultured in the presence of $1 \%$ nonessential amino acids (Biochrom AG, Berlin, Germany). Cells were transiently transfected with either Nanofectin (PAA, Pasching, Austria) or Nucleofection for Amaxa-electroporation (Amaxa/Lonza, Köln, Germany) in accordance with manufacturers' instructions. Transfections of different vector constructs were performed with adjusted molar amounts.

Single protein monitoring through multiparametric biosensing. The impact of single overexpressed sirtuin proteins (wtSIRT3, $\mathrm{SIRT}_{\mathrm{R} 80 \mathrm{~W}}$, SIRT3 ${ }_{\mathrm{V} 208 \mathrm{I}}$, wtSIRT1) on cellular metabolism was monitored subsequent to transient transfection of equimolar amounts of eukaryotic expression constructs into HeLa cells and real-time in vitro monitoring of glycolysis, respiration and cell adhesion with the Bionas biosensor chip system (Bionas, Rostock, Germany) (22).

Histone isolation. Histone proteins were extracted from 293T cells as previously published after incubation with $25 \mathrm{nM}$ Trichostatin A (TSA) (Sigma Aldrich, Deisenhofen, Germany) and $5 \mathrm{mM}$ nicotinamide (NAM) (Sigma Aldrich) (23). 
Immunoprecipitation and SIRT3 activity test via histone deacetylation assay. Flag tagged wtSIRT3, SIRT3 $3_{\mathrm{R} 80 \mathrm{w}}$, SIRT3 $_{\text {V208I }}$ were immunoprecipitated and used for in vitro deacetylation assays as previously described (11).

Protein stability analyses. Twenty-four hours after transient transfection of wtSIRT3, SIRT3 ${ }_{\mathrm{R} 80 \mathrm{w}}$ or SIRT3 ${ }_{\mathrm{V} 208 \mathrm{I}}$ HeLa cells were incubated with cycloheximide (Sigma Aldrich) in order to block protein synthesis for up to $48 \mathrm{~h}$. Protein samples were prepared at different time points and analyzed by Western blotting.

Western blotting. Western blots were performed according to standard protocols on nitrocellulose membranes (Biorad, Hercules, CA) and visualized by enhanced chemiluminescence (GE Healthcare, Buckinghamshire, UK).

Plasmids and antibodies. Eukaryotic expression constructs that have been used in this study: hSIRT3-Flag (GenBank AF083108, pcDNA3.1) (kindly provided by E. Verdin, The Gladstone Institute, San Francisco, CA) and hSIRT1-Flag (GenBank AF083106, pCMVtag4a; Gibco Invitrogen, Carlsbad, CA). Site-directed mutagenesis (QuikChange Mutagenesis Kit, Stratagene, Cedar Creek, TX) was carried out to generate the hSIRT3 $3_{\mathrm{H} 248 \mathrm{Y}}-\mathrm{FLAG}$, hSIRT3 $_{\mathrm{R} 80 \mathrm{w}}, \mathrm{hSIRT}_{\mathrm{V} 208 \mathrm{I}}$ constructs. SIRT3 wt, SNP and inactive mutants were further cloned into the pEGFP-C1 vector (Clontech Laboratories, Saint-Germain-en-Laye, France) via EcoRI restriction sites. All construct sequences were verified by direct DNA sequencing. Antibodies that were used for immunoblotting: antiFlag M2, anti-tubulin (all from Sigma-Aldrich), anti-SIRT3 (Imgenex, San Diego, CA), anti-c-Myc (own production), anti-H4 (ab31827; Abcam, Cambridge, UK) and antiH4-Lys16 (Upstate Biotechnology, Hamburg), anti-rabbit (Dianova, Hamburg), anti-mouse (Dianova).

Statistical analysis. SNPs were analyzed for concordance with the Hardy-Weinberg equilibrium with the online calculator of Michael H. Court (http://www.tufts.edu/ mcourt01).

\section{Results and Discussion}

Mitochondrial dysfunction is linked to a number of metabolic diseases, degenerative disorders and cancer. Based on the multifaceted mitochondrial functions of SIRT3, SIRT3 nSNPs could be of pathophysiological significance in this context.

Identification and validation of putative SIRT3 nSNPs. In silico analyses of three SNP databases [dbSNP (19), HapMap (20), and ABI (21)] for putative SIRT3 nSNPs (AF083108) allowed the identification of six candidate SNPs (Table I, Fig. 2), of which one SNP was located within a putative splice site (19).

SIRT3 $_{\text {R80W }}$ is localized at the N-terminal end, which is being processed after mitochondrial import; SIRT3 ${ }_{\mathrm{V} 208 \mathrm{I}}, \mathrm{SIRT}_{\mathrm{R} 214 \mathrm{G}}$ and SIRT3 $3_{\mathrm{T} 255 \mathrm{~N}}$ are part of the conserved active deacetylase domain and $\mathrm{SIRT}_{\mathrm{G} 369 \mathrm{~S}}$ is located within the SIRT3 C-terminus.

Validation of putative SIRT3 nSNPs from in silico data. Putative SIRT3 nSNPs (Table I) were analyzed for allelic

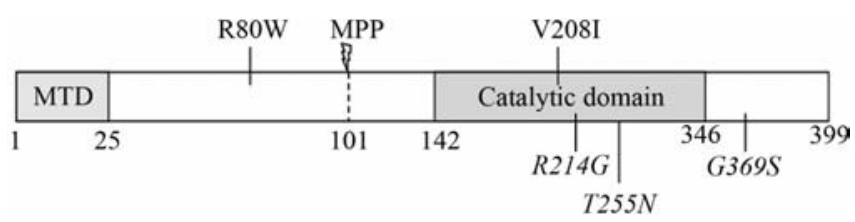

Figure 2. Identified and validated SIRT3 nSNPs localised to functional SIRT3 domains. SNPs identified as false positive in the Caucasian population are presented in italic letters. MTD, mitochondrial target sequence; MPP, matrix processing peptidase.

frequencies in 640 DNA samples from healthy donors by quantitative Taqman PCR SNP analysis and were then compared to database data (if available). While our analyses confirmed two nSNPs, SIRT3 ${ }_{\text {R80w }}$ and SIRT3 ${ }_{\mathrm{V} 2081}$, the other four candidate SNPs were identified to be either false positives or at least not present in Caucasians (Fig. 3) since they did not reveal any allelic variations.

Genotype frequency analyses for SNP SIRT $3_{\mathrm{R} 80 \mathrm{~W}}$ identified $72 \%$ to be homozygous $(\mathrm{C} / \mathrm{C}), 23.5 \%$ heterozygous $(\mathrm{C} / \mathrm{T})$ and $4.5 \%$ homozygous $(\mathrm{T} / \mathrm{T})$ for the wt and variant alleles respectively. At the beginning of our analyses the databases (dbSNP Build ID: build 126) gave only information about SIRT3 $3_{\text {R80W }}$ frequencies in people of African origin $(80.6 \% \mathrm{G} / \mathrm{G}, 19.4 \%$ $\mathrm{A} / \mathrm{G})$. In fact, for Caucasians, data on $\mathrm{SIRT} 3_{\mathrm{R} 80 \mathrm{~W}}$ are given in the HapMap database with the following frequencies: $71.7 \%$ wt, $26.5 \%$ heterozygous and $1.8 \%$ homozygous for variant allele, which is close to the data that have been generated in our laboratory.

For SIRT3 ${ }_{\mathrm{V} 208 \mathrm{I}}$ we revealed a genotype frequency of $60 \%$ homozygous $(\mathrm{G} / \mathrm{G}), 35 \%$ heterozygous $(\mathrm{G} / \mathrm{A})$ and $6 \%$ homozygous (A/A) for the wt and variant alleles, compared to 72,23 and $5 \%$ in the databases, respectively (20) (Fig. 3). However, the data that have been generated in our laboratory are statistically more reliable, since our sample size of 640 significantly exceeds the HapMap sample size of 113.

Our results on non-validated SIRT3 nSNPs are in accordance with HapMap data, revealing 100\% homozygous wt genotypes for false positive SNPs $(19,20)$. Genotype frequencies for $\operatorname{SIRT}_{\text {R80W }}(1.866)$ and $\operatorname{SIRT}_{\mathrm{V} 208 \mathrm{I}}(0.087)$ were both in accordance with the Hardy-Weinberg equilibrium ( $\mathrm{p}=0.172$ and 0.766 , respectively).

As a result of our study we found databases to give an incorrect picture for SIRT3 SNP allelic frequencies, since these are based on unvalidated information. The recent literature on the topic therefore offers not only insufficient but also quite inconsistent data on SIRT3 SNPs and their potential functional impact.

Rose et al postulated an impact of SIRT3 $\mathrm{SNP}_{\mathrm{G} 477 \mathrm{~T}}$ on survival in the elderly (24). This SNP did not lead to a change in the amino acid level (Ser159Ser) and it was shown by subsequent analyses by the authors themselves, that the putative impact of this SNP was due to a linkage disequilibrium with a VNTR (variable number of tandem repeats) allele specific enhancer located in intron 5 of SIRT3 (25). Since we had focussed our analyses on nSNPs and a recently reported meta-analysis on 2,461 samples by Lescai et al was not able to confirm the effect of this SNP or the VNTR allele specific enhancer, they were not considered in the context of this study (18). In addition, in that study no positive association 


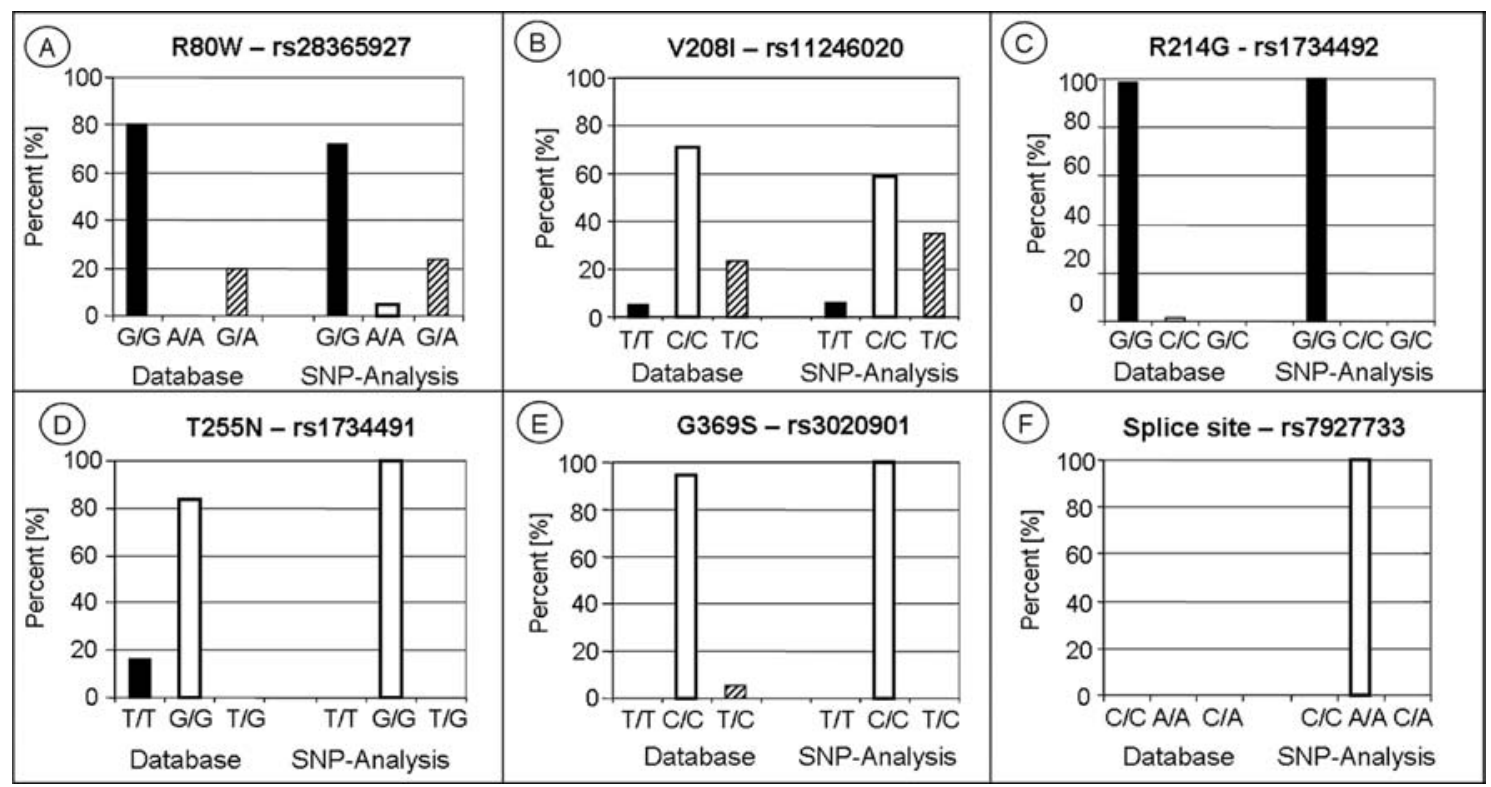

Figure 3. SIRT3 SNPs - distribution of allelic frequencies in 640 healthy donors.

was reported except for one SIRT3 SNP (rs939915), which we had, however, identified to be an SNP of the neighbouring gene psmd13, but unfortunately not a SIRT3 SNP.

\section{Functional analyses of validated SIRT3 nSNPs}

Impact of SIRT3 SNPs on protein activity. Both validated SIRT3 nSNPs, SIRT3 $3_{\text {R80w }}$ and SIRT3 ${ }_{\mathrm{V} 208 \mathrm{I}}$ go along with changes in the SIRT3 amino acid sequence and may affect SIRT3 protein levels and SIRT3 functional activity. Based on the SIRT3 histone deacetylase activity on histone $\mathrm{H}_{\mathrm{K} 16}(26)$, SIRT3 deacetylation activity was assessed for wtSIRT3 and its SNP mutants on hyperacetylated H4 histone proteins in order to further assess their impact on SIRT3 activity (Fig. 4A). Western blot analyses showed reduced signals of acetylated $\mathrm{H} 4_{\mathrm{K} 16}$ without significant differences for wtSIRT3 and the SIRT3 nSNPs. Incubation with the sirtuin specific inhibitor NAM as well as the SIRT3 inactive mutant completely abrogated both, wt and nSNPs SIRT3 deacetylase activity.

Impact of SIRT3 SNPs on protein stability. For SIRT3 stability analyses HeLa cells were transfected with SIRT3-flag vectors (wt, R80W, V208I). Twenty-four hours post transient transfection cells were incubated with the protein inhibitor CHX and analyzed for up to $72 \mathrm{~h}$ of $\mathrm{CHX}$ treatment by Western blotting (Fig. 4B). To visualize the effect of $\mathrm{CHX}$ we checked for $\mathrm{c}-\mathrm{Myc}$ protein levels since c-Myc has a short half-life (<30 min) (27). C-Myc signals were strongly reduced and absent after less than $2 \mathrm{~h}$ of $\mathrm{CHX}$ protein inhibition, while stable signals during treatment with the CHX solvent DMSO indicated that the solvent had no influence. In contrast to the rapid reduction of signals for c-Myc, the signals for the mitochondrial, mature SIRT3 (maSIRT3) remained constantly elevated with a first decrease after $34 \mathrm{~h}$ of $\mathrm{CHX}$ treatment.

Our stability analyses, which again showed no difference between wt and SNP mutants, offer the first evidence for SIRT3 stability. While the mean half-life of mitochondrial proteins in mammalian cells is around 3.5 to 5 days, for some proteins the half-lives are as short as 20 to $80 \mathrm{~min}$ (28). The SIRT3 half-life has been determined to be around 48-60 h, which appears to be rather short as it is ranges below the mean half-life for most mitochondrial proteins. Also, it appears to be more stable than it was predicted in silico (Expasy, ProtParam software), which anticipated a half-life of around $4.4 \mathrm{~h}$ for mammalian SIRT3 (29).

Monitoring of SIRT3 bioactivity. We further analyzed the influence of wtSIRT3, its SNP mutants and wtSIRT1 on cellular metabolism (Fig. 5) subsequent to transient transfection on a biosensor chip system, which gives the opportunity to analyse the effect of a specific protein on cell metabolism in living cells through real-time monitoring of metabolically relevant parameters, such as oxygen consumption, acidification rate and cell adhesion allowing conclusions towards cellular energy metabolism, specially respiration and glycolysis.

These analyses show that SIRT3 overexpression led to a significant increase of cellular respiration by $40 \%$, which was significantly stronger than the effect that was observed for an overexpression of SIRT1 (15\%). While SIRT3 overexpression had a strong impact on respiration, it had no influence on cellular adhesion or glycolysis (data not shown). In concordance with our results that were obtained in reference to the potential impact of SIRT3 SNPs on SIRT3 activity and stability, the effects of SIRT3 nSNPs on cellular metabolism were without relevance.

Our study is the first direct comparative analysis of SIRT3 and SIRT1 on cellular metabolism. The stronger increase of cellular respiration for SIRT3 may be based on the fact that SIRT3 has both indirect $(7,30)$ and direct activating effects on energy metabolism, especially respiration in contrast to SIRT1 (Fig. 1) (6). All these data help to explain the significant increase of respiration due to SIR3 overexpression and emphasize the important role of SIRT3 in cellular energy metabolism. Taking into account a transfection efficiency of 50-60\% in our experiments the observed increase 
(A)

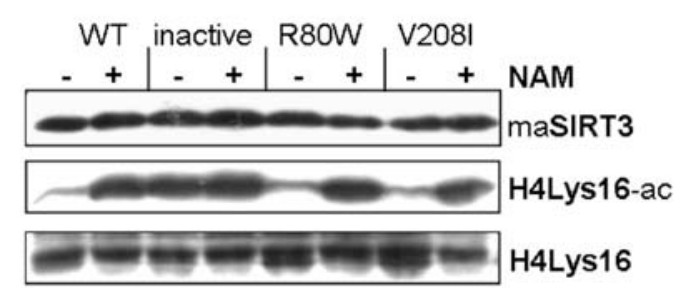

(B)
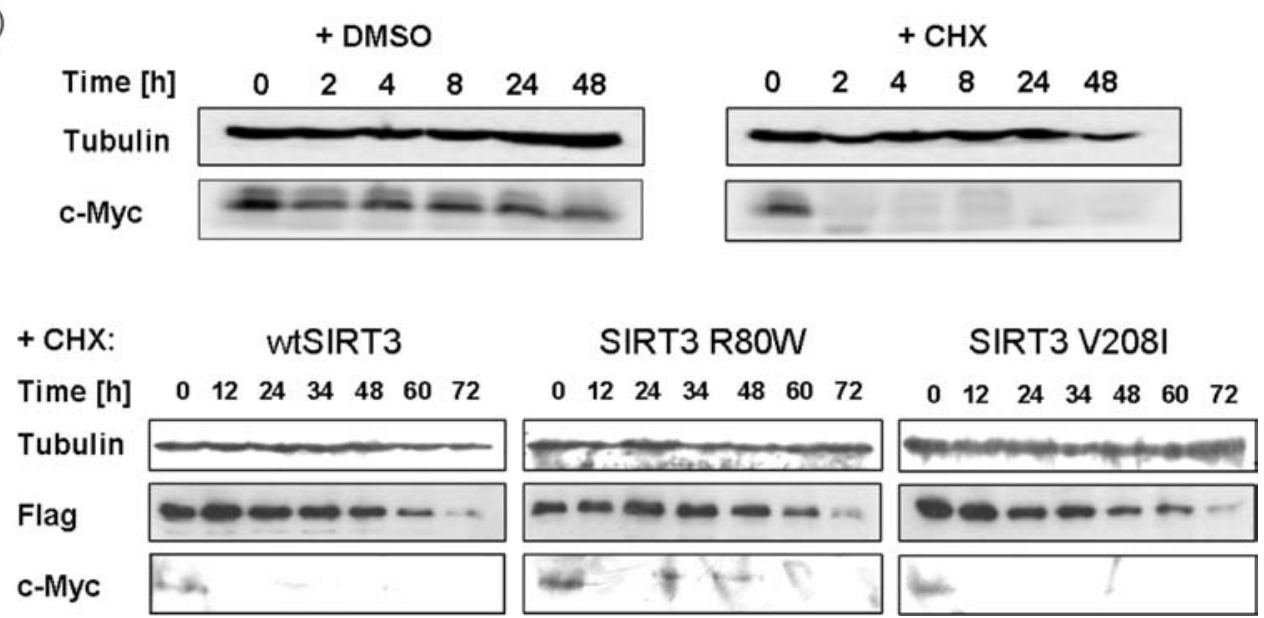

Figure 4. Influence of SIRT3 SNPs on SIRT3 activity and stability. (A) Histone deacetylation assay. Same amounts of purified hyperacetylated histones (H4) and wtSIRT3, SIRT3 ${ }_{\mathrm{H} 248 \mathrm{Y}}$ (inactive) SIRT3 ${ }_{\mathrm{R} 80 \mathrm{~W}}$ and SIRT3 ${ }_{\mathrm{V} 208 \mathrm{I}}$ were incubated for 20 min in HDAC-assay-buffer (with NAD) and with or without NAM. (B) SIRT3 stability assay. SIRT3 (wt, R80W, V208I) transfected cells were incubated with CHX, DMSO and kinetically analyzed via Western blotting for tubulin, c-Myc and maSIRT3 signals.

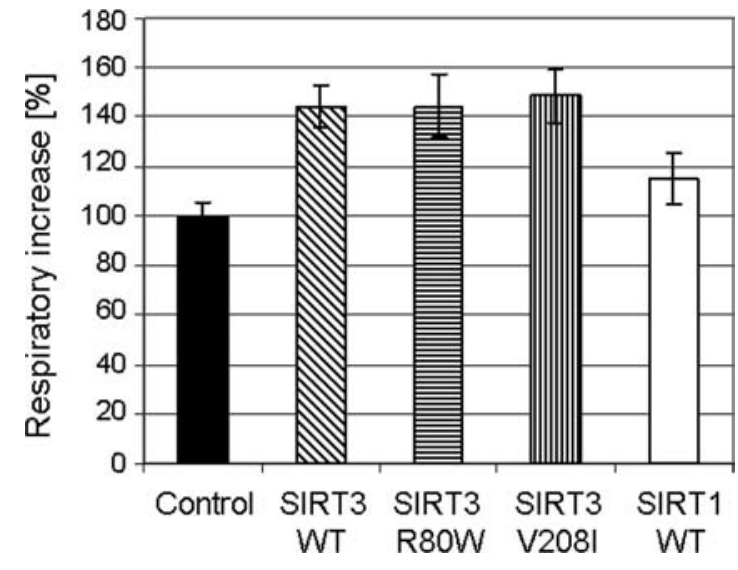

Figure 5. Influence of wtSIRT3 and functional impact of SIRT3 SNPs on cellular respiration in comparison to wtSIRT1. Control approaches were performed with empty vector, SIRT3 inactive mutant and without transfection, respectively.

of respiration of 40 and $15 \%$ did not reflect the full potential of SIRT3 and SIRT1, which may be up to around 80 and $30 \%$ respectively, assuming a transfection efficiency of $100 \%$. This would be in concordance with preliminary in vitro data showing an increase of respiration of $80 \%$ in HIB1B cells overexpressing mouse SIRT3 (7).

Possible explanations for the lack of impact of SIRT3 nSNPs. Despite their localization within the conserved deacetylase domain of SIRT3 and the identification of a potential glycosylation site in position aa 207-210 (31), the $\mathrm{SNP}_{\mathrm{V} 208 \mathrm{I}}$ was not expected to have a strong influence on protein levels since the change in the amino acid was between two amino acids of the same biochemical group.

$\mathrm{SNP}_{\mathrm{R} 80 \mathrm{~W}}$ results in a change from a positively charged, highly basic amino acid into an aromatic, neutral amino acid, which was anticipated to have functional impact. The lack of functional consequences of $\mathrm{SNP}_{\mathrm{R} 80 \mathrm{~W}}$ may in part be explained by its N-terminal localisation, which is most essentially processed after mitochondrial import. The postulated role of the N-terminus in nuclear localisation and function of SIRT3 seems not be affected either (8).

Taken together, our analyses provide the first evidence that SIRT3 is a rather stable protein, with a half-life of more than $48 \mathrm{~h}$ and reveal that SIRT3 has only two nSNPs, which however seem to have no functional impact. Furthermore, we show that in living cells SIRT3 has strong activating effects on cellular respiration which are significantly higher than the effect of SIRT1. These results further prompts the emerging importance of SIRT3 in the context of cell survival, metabolism, degenerative disease and cancer and point out that the impressive functions of SIR2 in model organisms (like yeast, Caenorhabditis elegans, and Drosophila melanogaster) can only be sufficiently assessed in humans in combinational analyses that consider all or at least the most important sirtuin isoforms, under which SIRT1 and SIRT3 are the most important candidates.

\section{Acknowledgements}

We thank E. Verdin (The Gladstone Institute, San Francisco, CA) for providing the expression vectors for hSIRT3 wt and N. Fadle for the c-Myc antibody. This study was supported by 
grants from the Deutsche José Carreras Leukämie-Stiftung e.V. (DJCLS R 05/11) to U.M.

\section{References}

1. Dali-Youcef N, Lagouge M, Froelich S, Koehl C, Schoonjans K and Auwerx J: Sirtuins: the 'magnificent seven', function, metabolism and longevity. Ann Med 39: 335-345, 2007.

2. Pallas M, Casadesus G, Smith MA, et al: Resveratrol and neurodegenerative diseases: activation of SIRT1 as the potential pathway towards neuroprotection. Curr Neurovasc Res 6: 70-81, 2009.

3. Michan S and Sinclair D: Sirtuins in mammals: insights into their biological function. Biochem J 404: 1-13, 2007.

4. Jiang WJ: Sirtuins: novel targets for metabolic disease in drug development. Biochem Biophys Res Commun 373: 341-344, 2008.

5. Lombard DB, Alt FW, Cheng HL, et al: Mammalian Sir2 homolog SIRT3 regulates global mitochondrial lysine acetylation. Mol Cell Biol 27: 8807-8814, 2007.

6. Ahn BH, Kim HS, Song S, et al: A role for the mitochondrial deacetylase Sirt3 in regulating energy homeostasis. Proc Natl Acad Sci USA 105: 14447-14452, 2008.

7. Shi T, Wang F, Stieren E and Tong Q: SIRT3, a mitochondrial sirtuin deacetylase, regulates mitochondrial function and thermogenesis in brown adipocytes. J Biol Chem 280: 13560-13567, 2005.

8. Scher MB, Vaquero A and Reinberg D: SirT3 is a nuclear $\mathrm{NAD}^{+}$-dependent histone deacetylase that translocates to the mitochondria upon cellular stress. Genes Dev 21: 920-928, 2007.

9. Sundaresan NR, Samant SA, Pillai VB, Rajamohan SB and Gupta MP: SIRT3 is a stress-responsive deacetylase in cardiomyocytes that protects cells from stress-mediated cell death by deacetylation of Ku70. Mol Cell Biol 28: 6384-6401, 2008.

10. Allison SJ and Milner J: SIRT3 is pro-apoptotic and participates in distinct basal apoptotic pathways. Cell Cycle 6: 2669-2677, 2007.

11. Schwer B, Bunkenborg J, Verdin RO, Andersen JS and Verdin E: Reversible lysine acetylation controls the activity of the mitochondrial enzyme acetyl-CoA synthetase 2. Proc Natl Acad Sci USA 103: 10224-10229, 2006.

12. Schlicker C, Gertz M, Papatheodorou P, Kachholz B, Becker CF and Steegborn C: Substrates and regulation mechanisms for the human mitochondrial sirtuins Sirt3 and Sirt5. J Mol Biol 382: 790-801, 2008

13. Jacobs KM, Pennington JD, Bisht KS, et al: SIRT3 interacts with the daf-16 homolog FOXO3a in the mitochondria, as well as increases FOXO3a dependent gene expression. Int J Biol Sci 4: 291-299, 2008.

14. Kim SC, Sprung R, Chen Y, et al: Substrate and functional diversity of lysine acetylation revealed by a proteomics survey. Mol Cell 23: 607-618, 2006.
15. Schwer B, North BJ, Frye RA, Ott M and Verdin E: The human silent information regulator (Sir)2 homologue hSIRT3 is a mitochondrial nicotinamide adenine dinucleotide-dependent deacetylase. J Cell Biol 158: 647-657, 2002.

16. Bradbury CA, Khanim FL, Hayden R, et al: Histone deacetylases in acute myeloid leukaemia show a distinctive pattern of expression that changes selectively in response to deacetylase inhibitors. Leukemia 19: 1751-1759, 2005.

17. Ashraf N, Zino S, Macintyre A, et al: Altered sirtuin expression is associated with node-positive breast cancer. $\mathrm{Br} \mathrm{J}$ Cancer 95: 1056-1061, 2006.

18. Lescai F, Blanche H, Nebel A, et al: Human longevity and 11p15.5: a study in 1321 centenarians. Eur J Hum Genet 17: 1515-1519, 2009.

19. Sherry ST, Ward MH, Kholodov M, et al: dbSNP: the NCBI database of genetic variation. Nucleic Acids Res 29: 308-311, 2001.

20. The International HapMap Project. Nature 426: 789-796, 2003.

21. De La Vega FM, Dailey D, Ziegle J, Williams J, Madden D and Gilbert DA: New generation pharmacogenomic tools: a SNP linkage disequilibrium Map, validated SNP assay resource, and high-throughput instrumentation system for large-scale genetic studies. Biotechniques 33 (Suppl): 48-54, 2002.

22. Thedinga E, Kob A, Holst H, et al: Online monitoring of cell metabolism for studying pharmacodynamic effects. Toxicol Appl Pharmacol 220: 33-44, 2007.

23. Engel $\mathrm{N}$ and Mahlknecht U: Aging and anti-aging: unexpected side effects of everyday medication through sirtuin 1 modulation. Int J Mol Med 21: 223-232, 2008.

24. Rose G, Dato S, Altomare K, et al: Variability of the SIRT3 gene, human silent information regulator Sir2 homologue, and survivorship in the elderly. Exp Gerontol 38: 1065-1070, 2003.

25. Bellizzi D, Rose G, Cavalcante P, et al: A novel VNTR enhancer within the SIRT3 gene, a human homologue of SIR2, is associated with survival at oldest ages. Genomics 85: 258-263, 2005.

26. Vaquero A, Sternglanz R and Reinberg D: NAD ${ }^{+}$-dependent deacetylation of H4 lysine 16 by class III HDACs. Oncogene 26: 5505-5520, 2007.

27. Escamilla-Powers JR and Sears RC: A conserved pathway that controls c-Myc protein stability through opposing phosphorylation events occurs in yeast. J Biol Chem 282: 5432-5442, 2007.

28. Russell SM, Burgess RJ and Mayer RJ: Protein degradation in rat liver. Evidence for populations of protein degradation rates in cellular organelles. Biochim Biophys Acta 714: 34-45, 1982.

29. Wilkins MR, Gasteiger E, Bairoch A, et al: Protein identification and analysis tools in the ExPASy server. Methods Mol Biol 112: 531-552, 1999

30. Hallows WC, Lee S and Denu JM: Sirtuins deacetylate and activate mammalian acetyl-CoA synthetases. Proc Natl Acad Sci USA 103: 10230-10235, 2006.

31. Onyango P, Celic I, McCaffery JM, Boeke JD and Feinberg AP: SIRT3, a human SIR2 homologue, is an NAD-dependent deacetylase localized to mitochondria. Proc Natl Acad Sci USA 99: 13653-13658, 2002. 